\title{
The vertical orbicularis oculi muscle turn-over procedure for the correction of paralytic ectropion of the lower eyelid
}

\author{
Ryuichi Azuma, Shimpo Aoki, Tetsushi Aizawa, Masahiro Kuwabara, Tomoharu Kiyosawa \\ Department of Plastic Surgery, National Defense Medical College, Tokorozawa, Japan
}

Background Static reconstruction surgery that tightens the tension of the inferior tarsus, thereby raising the lax lower eyelid, is a common treatment for paralytic ectropion of the lower eyelid. We present one such operative procedure, in which an orbicularis oculi muscle flap was used.

Methods The surgical technique involves partial resection of the tarsus and the skin, as well as a superior-based orbicularis oculi muscle flap that is sutured to the firm tissue present on the Whitnall tubercle. The muscle flap is approximately $7 \mathrm{~mm}$ in width and $15 \mathrm{~mm}$ in length, with a superior pedicle that is attached to the tarsus at the medial point of the resected tarsus. The procedure results in contact between the ocular surface and the lower eyelid.

Results The procedure was performed in 11 patients with lower eyelid ectropion due to facial paralysis. Ten cases showed a favorable outcome following surgery, with stable results seen over an average follow-up period of 4.5 years. In one case, recurrence of ectropion was observed 2 months after surgery due to an insufficient correction, and the patient required repeat surgery.

Conclusions The orbicularis oculi muscle flap was an effective means of suspension and was able to maintain long-term traction tension. This procedure can therefore be considered a favorable treatment option for lower eyelid ectropion due to facial paralysis.

Keywords Eyelids / Facial paralysis / Ectropion / Reconstructive surgical procedures
Correspondence: Ryuichi Azuma Department of Plastic Surgery, National Defense Medical College, 3-2 Namiki, Tokorozawa 359-0042, Japan

Tel: +81-4-2997-5156

Fax: +81-4-2997-5156

E-mail: azuma@ndmc.ac.jp

This article includes material presented at the 54th Annual Meeting of the Japan Society of Plastic and Reconstructive Surgery on April 13-15, 2011 in Tokushima, Japan.

Received: 28 Jul 2017 • Revised: 27 Dec $2017 \bullet$ Accepted: 20 Jan 2018

pISSN: 2234-6163 • elSSN: 2234-6171 • https://doi.org/10.5999/aps.2017.01235• Arch Plast Surg 2018;45:135-139

\section{INTRODUCTION}

Lower eyelid ectropion caused by facial paralysis results in keratoconjunctive disorders due to incomplete eyelid closure and cosmetic problems due to exposure of the palpebral conjunctiva [1]. Static reconstruction surgery, such as the lateral tarsal strip procedure, which raises the relaxed lower eyelid, is a common treatment for ectropion. In severe cases, fascia suspension is necessary to ensure sufficient tensile strength and permanence. We devised the vertical orbicularis oculi muscle turn-over (VOTO) procedure to effectively correct lower eyelid ectropion without requiring a fascia graft. The inferior tarsus is pulled postero-laterally and rotated inwardly with a muscle flap of the orbicularis oculi that is sutured to the Whitnall tubercle, and the skin and the orbicularis oculi muscle of the lower eyelid are resected so that the ectropion is effectively corrected. 


\section{METHODS}

\section{Subjects}

Eleven patients between 39 and 81 years of age (mean, 63.7 years), all with Asian eyelids, were treated between 2000 and 2015. Patients presented with relaxed lower eyelids, dry eye, or excessive lacrimation due to ectropion caused by chronic facial paralysis. The cause of the unilateral facial paralysis was acoustic neuroma in five cases, Bell palsy in two cases, Ramsay-Hunt syndrome in one case, maxillary cancer in one case, parotid gland cancer in one case, and squamous cell carcinoma of the cheek in one case. All patients provided written informed consent for the use of all clinical photographs for publication, which have been archived by the authors. The National Defense Medical College ethical committee approved this study on March 30, 2017 (No. 2707).

\section{Operative procedure}

The operative procedure was modified and developed over 15 years. The current procedure is presented below.

The degree of relaxation of the lower eyelid was assessed using a lateral traction test, wherein the lateral end of the lower eyelid was pinched by the fingers, pulled laterally, and the distance that the pinched portion moved was measured. The width of the inferior tarsus to be resected (typically 4-8 $\mathrm{mm}$ ) was calculated as half the length measured with the lateral traction test.

Local anesthesia was performed with $1 \%$ lidocaine containing 1:100,000 epinephrine injected into the lower lid and the lateral canthal region. A subciliary incision was made 2 -mm inferior to the gray line, extending laterally and slightly downward along a natural crease. The inferior tarsus and palpebral conjunctiva at the lateral angle of the eye were resected, based on the lateral traction test measurement, with a 2- to 3-mm margin remaining to suture the tarsus (Fig. 1A). An orbicularis oculi muscle flap (7-mm wide and 15-mm long) was created medial to the tarsus resection with a superior pedicle attached to the tarsus (Fig. 1B). Superficial fascia was included with the muscle flap in order to maintain its tensile strength. The lateral and medial stumps of the tarsus were sutured together with a single buried stitch, using 5-0 nylon. The conjunctiva was not sutured. The Whitnall tubercle was identified by blunt and blind dissection with mosquito forceps. The inferior tip of the orbicularis oculi muscle flap was turned over, pulled superiorly and laterally, and sutured to the firm tissue on the Whitnall tubercle using 5-0 nylon (Fig. 1B). The tension on the muscle flap was adjusted and slightly overcorrected. The traction of the orbicularis oculi muscle flap raised the tarsus superiorly, laterally, and posteriorly (with a "twisting effect") (Fig. 2), bringing the tarsus back into contact with the ocular surface. There was sufficient tensile strength in the muscle flap for it not to be torn apart or detached from the lid margin. The residual orbicularis oculi muscle stump (harvesting site) was sutured horizontally with three stitches using a 5-0 absorbable material, although the residual muscle was not sutured in the first four cases. A triangular piece of skin was resected laterally to the tarsus resection (Fig. 1A) and the remaining skin was trimmed as necessary and sutured (Fig. 1B).

\section{RESULTS}

The postoperative observation period ranged from 1 to 9.5 years, with an average of 4.5 years. In all cases, the lower eyelid ectropion was corrected after surgery, with significant improve-

\section{Fig. 1. Operative procedure}

(A) Resection of the tarsus and the conjunctiva was planned with a width of 4 to $8 \mathrm{~mm}$ and the orbicularis oculi muscle flap was prepared with a width of $7 \mathrm{~mm}$ and length of $15 \mathrm{~mm}$. The skin resection was placed laterally for cosmetic purposes. (B) The orbicularis oculi muscle flap was created with a superior pedicle medial to the tarsus resection. The muscle flap was rotated and turned upward and laterally. Four suturing procedures exerted an additive effect: one joined the lateral and medial stumps of the tarsus, one attached the tip of the orbicularis oculi muscle flap to the Whitnall tubercle, one secured the residual orbicularis oculi muscle, and one was used for the skin resection.

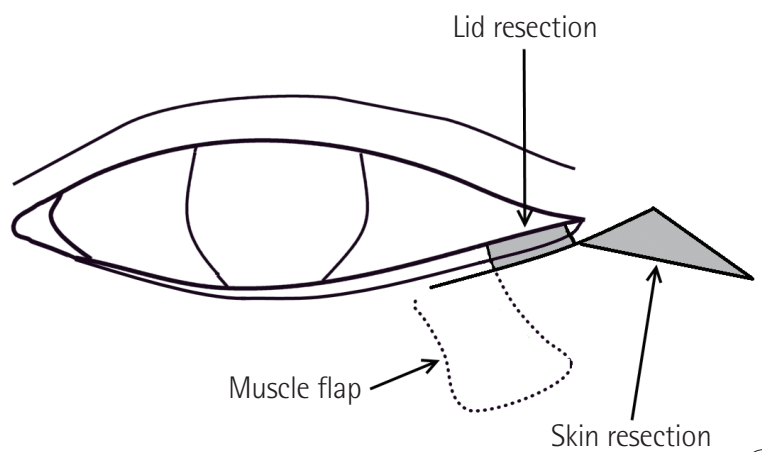

(A)

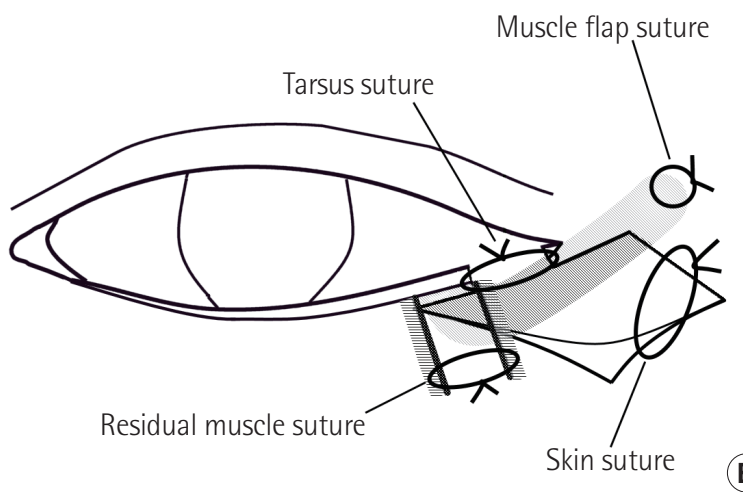


ment of symptoms such as xerophthalmia. The mean decrease ( \pm standard deviation) in the marginal reflex distance-2 (MRD2 ), which was measured before the operation and at the end of the follow-up period, was $2.1 \pm 0.7 \mathrm{~mm}$. In one case, insufficient correction caused a relapse 2 months after the procedure. Surgery was repeated 1 year later, with the use of a fascial graft. No other complications were observed within the observation period. Details of the cases are presented in Table 1.

\section{Case study}

A 61-year-old woman was referred to our hospital for paralytic lagophthalmos and scleral show 5 years after developing Bell palsy. The VOTO procedure was performed under local anesthesia, correcting the ectropion. No recurrence of symptoms was observed for 3 years after surgery. The MRD-2 decreased from $8.3 \mathrm{~mm}$ (pre-surgery) to $5.8 \mathrm{~mm}$ (post-surgery) (Fig. 3).

Another example is given in Fig. 4.

\section{Fig. 2. Mechanics of the procedure}

The black arrow indicates the direction of traction of the orbicularis oculi muscle flap, showing the twisting effect. The orbicularis oculi muscle flap was first turned up, then the inferior side of the tarsus was pulled up, and the tarsus was rotated internally.

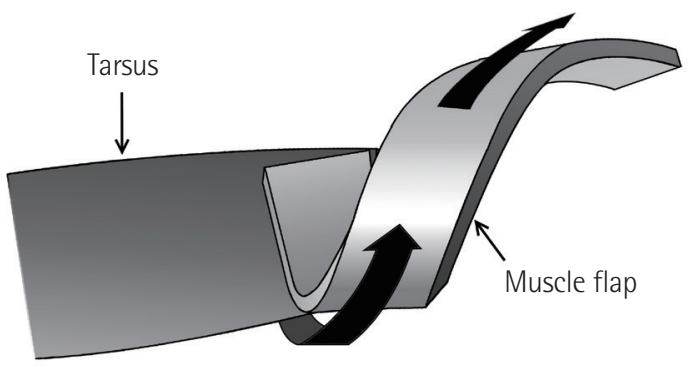

\section{Fig. 3. Case report 1}

(A) A 61-year-old woman presented with paralytic ectropion due to Bell palsy (preoperative view). (B) The resection width of the tarsus was $6 \mathrm{~mm}$, and the orbicularis oculi muscle flap was $6 \mathrm{~mm}$ wide and $13 \mathrm{~mm}$ long. (C) Two months after surgery. (D) Three years after surgery.
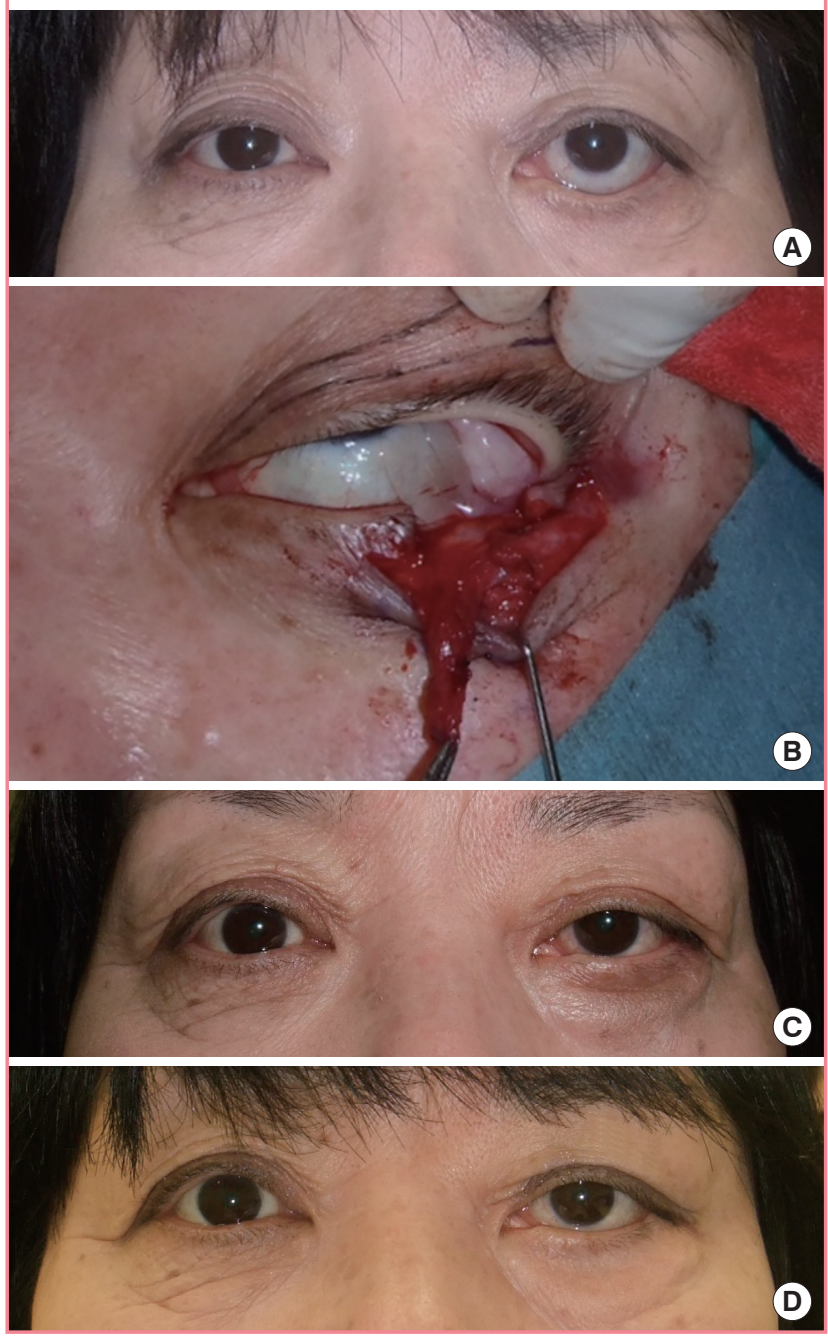

Table 1. Clinical characteristics of the participants

\begin{tabular}{|c|c|c|c|c|c|c|}
\hline No. & Age $(y r) / s e x$ & Primary disease & $\begin{array}{l}\text { MRD-2 pre-op } \\
(\mathrm{mm})\end{array}$ & $\begin{array}{l}\text { MRD-2 post-op } \\
(\mathrm{mm})^{\mathrm{a})}\end{array}$ & $\begin{array}{l}\text { Follow-up period } \\
\text { (mo) }\end{array}$ & Note \\
\hline 1 & $66 / F$ & Acoustic neuroma & 7.2 & 5.3 & 22 & \\
\hline 2 & $39 / M$ & Acoustic neuroma & 6.6 & 5.5 & 96 & \\
\hline 3 & $71 / F$ & Bell palsy & 9.4 & 6.2 & 33 & \\
\hline 4 & $50 / F$ & Ramsay-Hunt syndrome & 6.0 & 4.5 & 72 & \\
\hline 5 & $64 / \mathrm{M}$ & Parotid cancer & 7.5 & 5.3 & 75 & Fascia grafted afterward \\
\hline 6 & $62 / \mathrm{M}$ & Acoustic neuroma & 6.1 & 5.2 & 82 & \\
\hline 7 & $57 / F$ & Acoustic neuroma & 6.3 & 4.4 & 112 & \\
\hline 8 & $75 / \mathrm{M}$ & Maxillary cancer & 8.8 & 6.6 & 12 & \\
\hline 9 & $62 / F$ & Bell palsy & 8.3 & 5.8 & 23 & Case report 1 (Fig. 3) \\
\hline 10 & $81 / M$ & Skin cancer & 7.4 & 4.7 & 42 & \\
\hline 11 & $74 / \mathrm{M}$ & Acoustic neuroma & 8.6 & 6.0 & 25 & Case report 2 (Fig. 4) \\
\hline
\end{tabular}




\section{Fig. 4. Case report 2}

(A) A 74-year-old man developed paralytic lagophthalmos 2 years after resection of a left acoustic neuroma (preoperative view). (B) The outlined resection width of the tarsus was $6 \mathrm{~mm}$, and the size of the orbicularis oculi muscle flap was $7 \mathrm{~mm}$ wide and $15 \mathrm{~mm}$ high, and skin resection was performed laterally (blue arrows). (C) Orbicularis oculi muscle flap. (D) After surgery. (E) Three years after surgery.
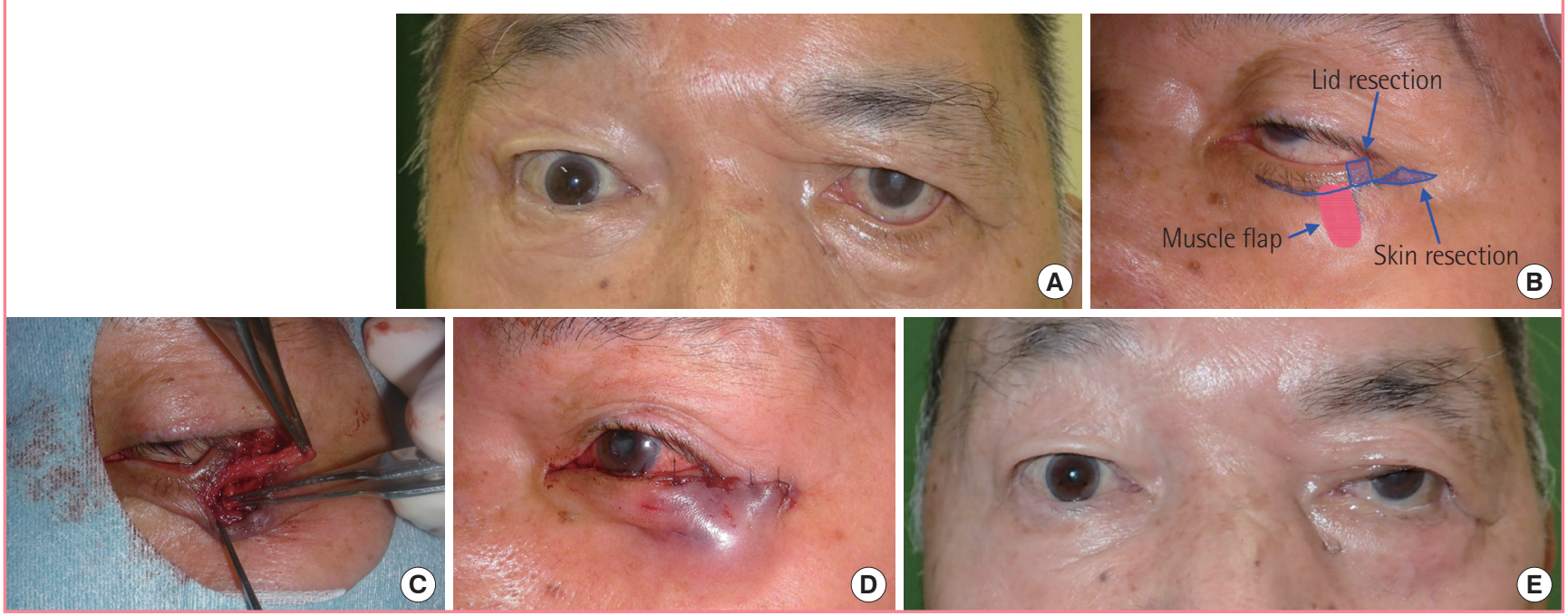

\section{DISCUSSION}

Contact between the ocular surface and the lower eyelid is lost in cases of ectropion of the lower eyelid, causing exposure of the palpebral conjunctiva and symptoms such as xerophthalmia, excessive lacrimation, and keratoconjunctivitis. There are three main types of ectropion: involutional ectropion, cicatricial ectropion, and paralytic ectropion. In Asian patients, lower eyelid ectropion is commonly caused by facial paralysis, and rarely occurs due to age-related laxity of the lower eyelid [2].

Operative procedures to correct lower eyelid ectropion due to facial paralysis include the Kuhnt-Szymanowski procedure [3], lateral canthopexy [4], and the tarsal strip procedure [5-7] in mild cases, and the temporalis muscle transposition $[8,9]$ in severe cases. The first 3 procedures are static reconstruction techniques. The temporalis muscle transposition is a dynamic reconstruction technique that allows eye closure when chewing, as the eyelids close on contraction of the temporalis muscle. The lower eyelid is less mobile than the upper eyelid and there is little need for dynamic reconstruction; therefore, the objective of lower eyelid ectropion surgery is to provide contact between the tarsus and ocular surface in a static state. Lateral canthopexy and the lateral tarsal strip procedure are widely performed and involve the correction of ectropion via resection and contraction of the tarsus; in these procedures, the direction of traction of the tarsus is parallel to the tarsus. The ocular surface is spherical, and in order to maximize the contact between the lower eyelid and the ocular surface, it is ideal to generate traction by pulling the lower eyelid superiorly and posteriorly and to generate torque to invert the tarsus, as performed in the VOTO procedure (Figs. 1B, 2).

Surgical procedures using an orbicularis oculi muscle flap to lift the lower eyelid have been reported by d'Alcontres et al. [10] (using the orbicularis oculi muscle of the upper eyelid) and by Sevim et al. [11] (using the horizontal orbicularis oculi muscle of the lower eyelid for prophylactic lid suspension in zygomatic fracture fixation). Using the VOTO procedure described in the present study, a strong ectropion correction effect was obtained by suturing and contracting the harvest site in the orbicularis oculi muscle flap; in addition, this procedure generated torque on the tarsus. This "twisting effect," which was not observed in the procedures described by d'Alcontres et al. [10] and Sevim et al. [11], brings the tarsus back into contact with the ocular surface. In the long term, drooping frequently occurs after lowereyelid reconstruction, due to gravity. It is thus important in static reconstruction to use a reconstruction material that will not become lax over time. An important consideration is whether the orbicularis oculi muscle flap has sufficient and permanent traction tension. In the VOTO procedure, although the direction in which the muscle flap is pulled differs from the direction of the muscle fibers, our surgical experience showed that the muscle flap had sufficient strength and did not tear even if strong traction was applied using 5-0 nylon. In this study, retrogression of the eyelid tension occurred 1 to 2 months following surgery, but not subsequently (with a maximum follow-up period of 9 years). Another surgical case, which was excluded from this study, involved the use of subcutaneous scar tissue as the reconstruction material, but ectropion recurred because the tissue be- 
came lax. Scar tissue does not provide the long-term tractive power that can be obtained through the orbicularis oculi muscle flap.

The VOTO procedure requires approximately 20 additional minutes to create and suture a muscle flap, compared to a simple canthoplasty. However, based on our experiences, it has a stronger effect and longer durability in ectropion correction compared to simple canthoplasty or the lateral tarsal strip technique. In cases of excessive relaxation of the eyelid, we consider the VOTO procedure to be advantageous compared to fascia suspension techniques [12], due to its simpler operative technique with an equivalent or greater effect.

\section{NOTES}

\section{Conflict of interest}

No potential conflict of interest relevant to this article was reported.

\section{Ethical approval}

The study was approved by the National Defense Medical College ethical committee (No. 2707) and performed in accordance with the principles of the Declaration of Helsinki. Written informed consents were obtained.

\section{Patient consent}

The patients provided written informed consent for the publication and the use of their images.

\section{ORCID}

Ryuichi Azuma https://orcid.org/0000-0002-7479-1779

\section{REFERENCES}

1. Rahman I, Sadiq SA. Ophthalmic management of facial nerve palsy: a review. Surv Ophthalmol 2007;52:121-44.
2. Carter SR, Chang J, Aguilar GL, et al. Involutional entropion and ectropion of the Asian lower eyelid. Ophthal Plast Reconstr Surg 2000;16:45-9.

3. Smith B, Cherubini TD. Oculoplastic surgery: a compendium of principles and technique. Saint Louis: C.V. Mosby Company; 1970.

4. Flowers RS, Duval C. Blepharoplasty and periorbital aesthetic surgery. In: Aston SJ, Beasley RW, Thorne CH, et al., editors. Grabb and Smith's plastic surgery. 5th ed. New York: Lippincott Williams \& Wilkins; 1997. p. 628-30.

5. Anderson RL, Gordy DD. The tarsal strip procedure. Arch Ophthalmol 1979;97:2192-6.

6. Becker FF. Lateral tarsal strip procedure for the correction of paralytic ectropion. Laryngoscope 1982;92:382-4.

7. Jordan DR, Anderson RL. The lateral tarsal strip revisited: the enhanced tarsal strip. Arch Ophthalmol 1989;107:6046.

8. Andersen JG. Surgical treatment of lagophthalmos in leprosy by the Gillies temporalis transfer. Br J Plast Surg 1961;14: 339-45.

9. Frey M, Giovanoli P, Tzou CH, Kropf N, Friedl S. Dynamic reconstruction of eye closure by muscle transposition or functional muscle transplantation in facial palsy. Plast Reconstr Surg 2004; 114:865-75.

10. d'Alcontres FS, Cuccia G, Lupo F, et al. The orbicularis oculi muscle flap: its use for treatment of lagophthalmos and a review of its use for other applications. J Plast Reconstr Aesthet Surg 2010;63:416-22.

11. Sevim KZ, Akcal A, Dagdelen D, et al. Beneficial effects of turnover orbicularis oculi muscle suspension flap for treating facial fractures via subciliary incision. J Craniofac Surg 2014;25:1465-7.

12. Terzis JK, Kyere SA. Minitendon graft transfer for suspension of the paralyzed lower eyelid: our experience. Plast Reconstr Surg 2008;121:1206-16. 